\title{
Effects of myofiber isolation technique on sarcolemma biomechanics
}

Karla P Garcia-Pelagio', Stephen JP Pratt ${ }^{2}$ \& Richard M Lovering*,2,3 iD

${ }^{1}$ Departmento de Física, Facultad de Ciencias, Universidad Nacional Autónoma de México, Mexico City, Mexico; ${ }^{2}$ Department of Physiology, University of Maryland School of Medicine, Baltimore, MD 21201, USA; ${ }^{3}$ Department of Orthopedics, University of Maryland School of Medicine, Baltimore, MD 21201, USA; *Author for correspondence: rlovering@som.umaryland.edu

BioTechniques 69: 00-00 (November 2020) 10.2144/btn-2020-0087

First draft submitted: 12 June 2020; Accepted for publication: 10 September 2020; Published online: 1 October 2020

\section{ABSTRACT}

Isolated myofibers are commonly used to understand the function of skeletal muscle in vivo. This can involve single isolated myofibers obtained from dissection or from enzymatic dissociation. Isolation via dissection allows control of sarcomere length and preserves tendon attachment but is labor-intensive, time-consuming and yields few viable myofibers. In contrast, enzymatic dissociation is fast and facile, produces hundreds of myofibers, and more importantly reduces the number of muscles/animals needed for studies. Biomechanical properties of the sarcolemma have been studied using myofibers from the extensor digitorum longus, but this has been limited to dissected myofibers, making data collection slow and difficult. We have modified this tool to perform biomechanical measurements of the sarcolemma in dissociated myofibers from the flexor digitorum brevis.

\section{METHOD SUMMARY}

Modifications of the elastimetry technique allowed us to obtain biomechanical measurements of the sarcolemma in enzymatically dissociated myofibers at a much higher throughput.

\section{KEYWORDS:}

cell mechanics • dissection • elastimetry • enzymatically dissociated fibers • myofibers

Dissection of single muscle fibers (myofibers) provides a method of isolating individual, viable myofibers with tendon on each end and control of sarcomere length (SL), but this method is labor-intensive and time-consuming, requiring a large sample size of whole muscles due to the low myofiber yield (Table 1). Single isolated myofibers can also be obtained by enzymatic dissociation of whole muscle, an established method that is facile and yields hundreds of intact myofibers for study; however, the tendons are digested in the process, and therefore myofiber length cannot be controlled. Sarcolemma biomechanics has been studied using dissected myofibers from the extensor digitorum longus (EDL) (Figure 1A), but we recently adapted the classical micropipette aspiration technique based in elastimetry methods [1-3] to study sarcolemma biomechanics using enzymatically isolated and plated myofibers from the flexor digitorum brevis (FDB) (Figure 1B).

Myofibers in healthy skeletal muscle are typically long and cylindrical in shape but can be malformed (Figure 2A \& B), which happens with much greater frequency in dystrophic and aging muscles than in healthy muscles [5-8]. The ability to assess the biomechanics of the sarcolemma in these malformed (e.g., branched) myofibers was the driving force behind using enzymatically dissociated myofibers, as dissecting intact branched myofibers tendon-to-tendon is unattainable. Enzymatic dissociation not only provides an abundance of myofibers in a short period of time, but also preserves those with malformed/branched morphology. The FDB muscle is typically used for enzymatic dissociation because its myofibers are short enough to easily plate in culture; as a result, cultures of FDB myofibers have been very practical, useful and widely used for morphological and physiological studies [5,6,9,10]. Here we describe the methods to test sarcolemma biomechanics on dissected versus dissociated myofibers and report some representative results, and necessary modifications, needed to adapt the dissection technique.

Age-matched male control mice (wild type [WT]) and dystrophic mice (MDX) from the C57BL/10ScSnJ strain (The Jackson Laboratory, ME, USA) were used for studies. The MDX mouse, a mouse model for the human disease Duchenne muscular dystrophy, was selected because myofibers have a variety of shapes, and mechanics of the sarcolemma are of great interest due to the absence of the sarcolemma-associated protein dystrophin. All experimental procedures were approved by the University of Maryland Institutional Animal Care \& Use Committee. Following euthanasia, FDB muscles were harvested bilaterally from WT and MDX mice. Single myofibers were enzymatically isolated in DMEM with $0.2 \%$ bovine serum albumin, $1 \mu \mathrm{l} / \mathrm{ml}$ gentamicin and $2 \mathrm{mg} / \mathrm{ml}$ type I collagenase for $1-3 \mathrm{~h}$ as 


\section{Table 1. Comparison of methods to measure sarcolemma biomechanics.}

Dissection Enzymatic dissociation

Sarcomere length Can adjust myofiber length and therefore sarcomere as needed

Cannot control myofiber length. Values of biomechanical properties Accurate measure of biomechanical properties depend on stiffness of cultured matrix used

Yield $\quad 1-2$ myofiber/muscle

Direct time $\quad 1-2 \mathrm{~h} \quad 30 \mathrm{~min}$

Hundreds myofibers/muscle

Skill Labor-intensive, and experience/good dissection technique required to Facile and minimal special skills required to obtain intact myofibers obtain intact single myofibers

Cost $\$ \$ \$$

Other Need to use a muscle with easily stabilized tendon at either end (e.g., extensor digitorum longus, TA). This also allows testing of contractile force

Limited to small muscles with relatively short myofibers. Digestion of tendons makes contractile testing difficult
(A)

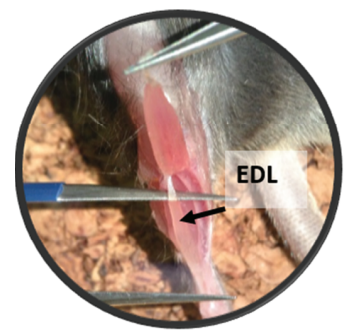

Whole muscle
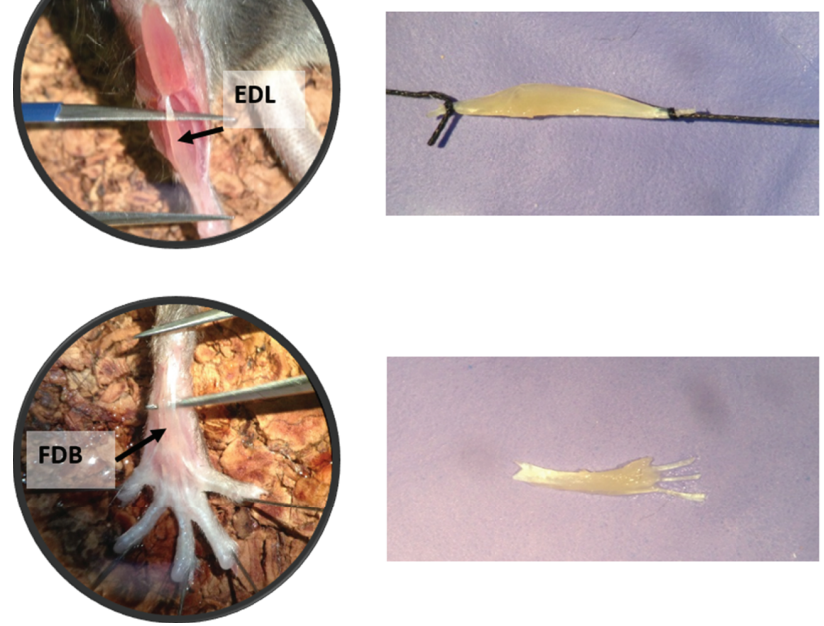

Single myofiber
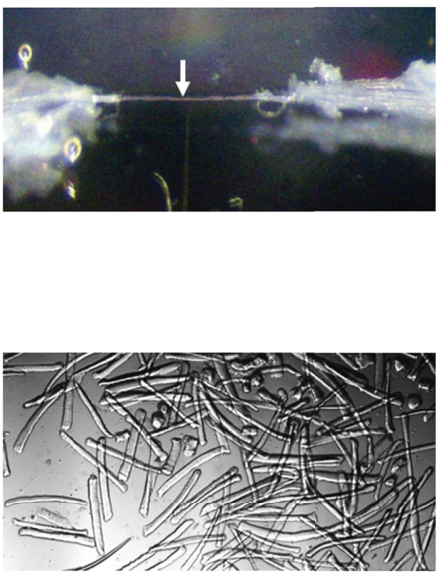

Figure 1. Methods for obtaining single isolated fibers. (A) The extensor digitorum longus muscle is shown in place (left), then after removal the muscle is tied at both tendons (middle) and myofibers are dissected away until a single myofiber remains (right). (B) The flexor digitorum brevis is shown in place (left). Once removed (middle), the flexor digitorum brevis muscle is placed into solution where isolated myofibers are obtained via enzymatic dissociation (right). Note that the dissection methods yield only one or two myofibers per muscle, whereas the enzymatic dissociation provides hundreds of isolated myofibers (which can be diluted as needed).

Adapted with permission from [4].

described previously [5,11]. Myofibers were then plated on extracellular matrix-coated imaging dishes. All of the myofibers were imaged and/or tested within a 24-h period.

Sarcolemma stiffness, Young's modulus, strain, compliance and viscoelasticity in single isolated myofibers can be measured using an established method called elastimetry $[3,4,9,12]$. Testing the mechanical properties of the sarcolemma via elastimetry has been used on single dissected myofibers [4] but has not been applied to dissociated myofibers until recently [9] and methods not compared until now. We used elastimetry to compare sarcolemma membrane fragility and compliance on dissected EDL myofibers and enzymatically dissociated FDB myofibers of WT and MDX mice. A borosilicate micropipette forming a vacuum, attached to a manometer, is placed on the surface of the myofiber, and a bleb is formed when negative suction pressure $(P)$ is applied and can be used to assess the elastic behavior of the sarcolemma. One can analyze the elastic behavior of the distortion, and tension lines formed by myofibrils, of the membrane in response to suction pressures applied over a small area as a bleb is formed (Figure 2C). Pressure exerted to the outside surface of the myofiber membrane is calculated from the hydrostatic pressure equation $\mathrm{P}=\rho g \mathrm{~h}_{\text {man }}$ in dynes $/ \mathrm{cm}^{2}, \mathrm{where} \rho=\operatorname{the}$ manometer's fluid density in grams per cubic centimeter, $g=981 \mathrm{~cm} / \mathrm{s}^{3}$, and $\mathrm{h}$ is the difference of levels in the manometer (cm) [12]. After bleb formation, larger increases in $P$ sever the connections between the sarcolemma and myofibrils $\left(P_{\text {separation }}\right)$ and eventually causes rupture of the cell membrane (membrane bursting, $\left.P_{\text {burst }}\right)$. Maximal pressure needed to cause disruption $\left(P_{\text {separation }}\right.$ and $\left.P_{\text {burst }}\right)$ is recorded and can be used to compare sarcolemma stability between groups of myofibers $[4,9]$. 
(A)

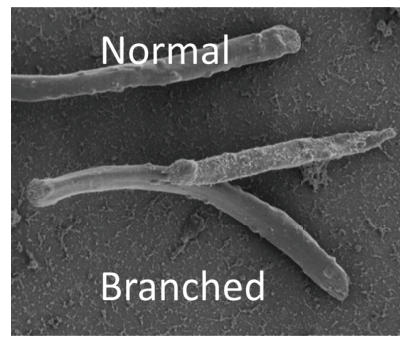

(B)

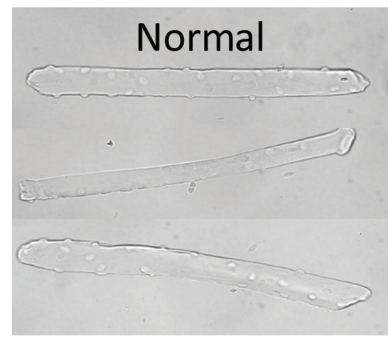

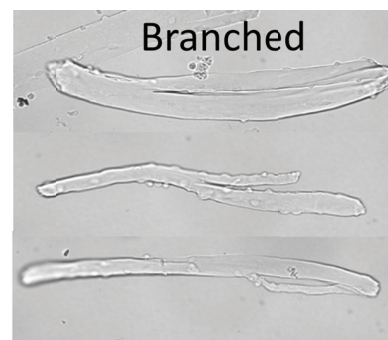

(C)
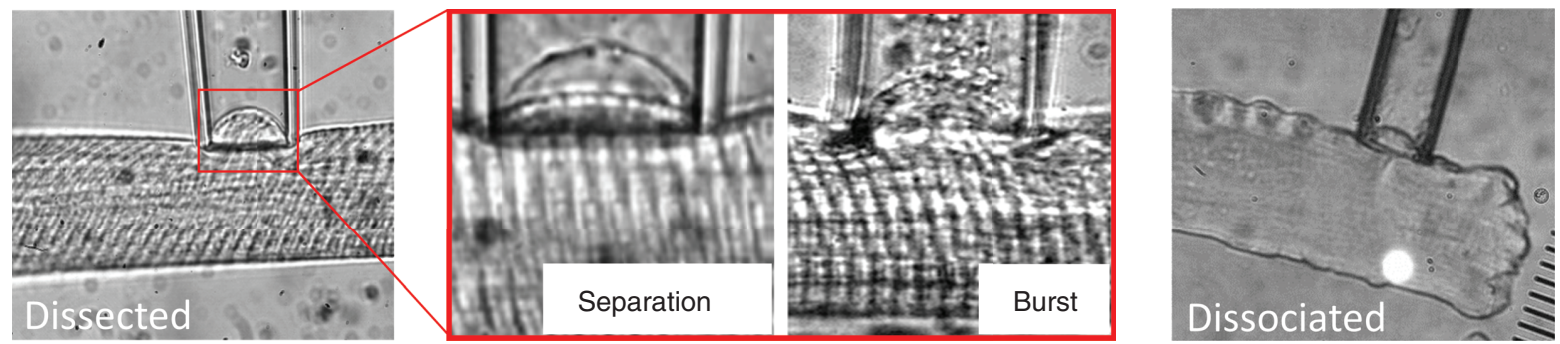

(D)
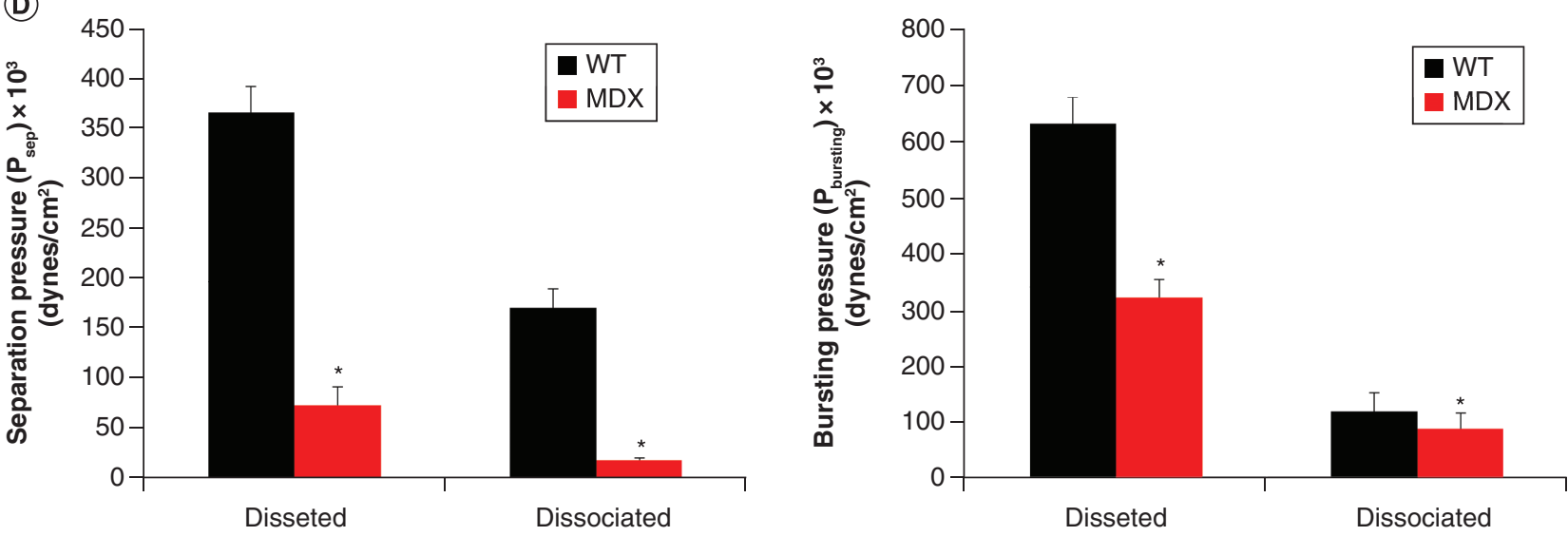

Figure 2. Elastimetry. (A) Scanning electron microscopy image shows examples of normal myofibers and branched myofibers with abnormal morphology of MDX mice. (B) Light microscopy shows further examples of such myofibers after enzymatic dissociation. Obtaining isolated myofibers with branched abnormal morphology is improbable, if not impossible, and therefore not able to be studied by the myofiber dissection technique. (C) Light microscopy image showing how an open-mouthed pipette is attached to a single myofiber to assess sarcolemma mechanical properties. Suction pressure (P) applied through a pipette to the sarcolemma form a bleb; bleb height increases with increasing P. Higher $\mathrm{P}$ causes the separation of the sarcolemma from myofibrils and eventual bursting of the sarcolemmal bleb. A sarcolemmal bleb inside a pipette can be obtained using either the dissection technique or enzymatic dissociation (far right panel). (D) Effect of dissected versus enzymatically dissociated myofibers in wild-type and MDX myofibers. Mean sarcomere length was $3.6 \pm 0.1$ and $3.2 \pm 0.1 \mu \mathrm{m}$ in dissected and dissociated myofibers, respectively. Observe that the values from enzymatic dissociation are up to six-times smaller compared with the dissected method. Note that the enzymatic method must be plated in a matrix to be tested. Statistical significance was assessed with a one-way ANOVA. $\mathrm{n}=8$ myofibers per group. Data presented as mean \pm SD. ${ }^{*} \mathrm{p}<0.05$.

Adapted with permission from [4].

Typically, elastimetry is only performed on dissected single myofibers suspended by clamping the tendons at either end [4,13]. To perform this method on dissociated myofibers where the tendons are not available, the cells must be plated in culture dishes. Furthermore, it is necessary to shave down the wall height of the culture dish for free access of the pipette. Because the myofibers are adhered to the coating of the glass-bottomed dish, myofiber length cannot be controlled, and this needs to be considered when examining sarcolemma biomechanics with this method (see Table 1). Although length cannot be adjusted in plated myofibers to match to length in dissected myofibers, SL can still be measured indirectly via light microscopy, as sarcolemma mechanics is affected by this value [4]. The pressure measurements we obtained from dissected EDL myofibers (where length is set) and dissociated FDB myofibers (where the cells can sit at varying lengths) varied in part likely due to unequal SL, but still yield important relative differences between WT and MDX myofibers 
using both methods (Figure 2D) [4,9]. When employing this technique, one should consider all potential confounding variables, such as muscle type, myofiber length, attachment to substrates and tendons when interpreting results.

Sarcolemma mechanics of isolated muscle fibers can be assessed using dissected myofibers or enzymatically dissociated myofibers, providing important flexibility in the method. Muscles such as the EDL or TA muscle can be used for dissection, and the EDL or FDB are both amenable to dissociation. Variable SLs, the use of different muscles, enzymatic dissociation and the required plating for dissociated myofibers all need to be considered. Indeed, the latter could underlie the difference in magnitude of recorded pressures between dissected and dissociated myofibers. For example, certain cell types can modify their internal stiffness to match the mechanical characteristics of their external substrates [14] and substrates can promote intracellular cell force generation [15], biological variables that can affect measured mechanical values. However, differences in measured magnitudes could also be due to the use of myofibers from different muscles or unequal SL due to methodology. Nevertheless, the ability to use elastimetry on enzymatically dissociated myofibers shows that the relative changes between WT and MDX are similar, and this method of using dissociated myofibers can provide much higher throughput for assessment of the sarcolemma strength in various disease models.

In summary, to study the biomechanical properties of the sarcolemma, we utilize a method - the elastimetry - whereby suction pressures applied through a pipette to the sarcolemma generate a deformation (a bleb) that is proportional in size to changing pressure. Large increases in pressure allow assessment of failure (burst) between the sarcolemma and underlying structures. Studies have only been performed on single dissected myofibers, a labor-intensive process with low throughput but allowing control of SL. Here we describe basic modifications to this method to include assessment of the sarcolemma in enzymatically dissociated plated myofibers, a much faster and facile approach. Such tools can hopefully provide a deeper understanding of myofiber health and disease, as well as application for other cell types.

\section{Author contributions}

KP Garcia-Pelagio and RM Lovering contributed to the conception and design. KP Garcia-Pelagio and SJP Pratt contributed to the acquisition and analysis of data. All authors contributed to the interpretation of the results and to manuscript writing.

\section{Financial \& competing interests disclosure}

This work was supported by grants to RM Lovering (R01AR059179) from the National Institutes of Health National Institute of Arthritis and Musculoskeletal and Skin Diseases, and by UNAM (PAPIIT IA 210320) and CONACyT (A1-S-17636) to KP Garcia-Pelagio. The authors have no other relevant affiliations or financial involvement with any organization or entity with a financial interest in or financial conflict with the subject matter or materials discussed in the manuscript apart from those disclosed.

No writing assistance was utilized in the production of this manuscript.

\section{Open access}

This work is licensed under the Attribution-NonCommercial-NoDerivatives 4.0 Unported License. To view a copy of this license, visit http://creativecommons.org/licenses/by-nc-nd/4.0/

\section{References}

1. Rand RP, Burton AC. Mechanical properties of the red cell membrane. I. Membrane stiffness and intracellular pressure. Biophys. J. 4(2), 115-135 (1964).

2. Gonzalez-Bermudez B, Guinea GV, Plaza GR. Advances in micropipette aspiration: applications in cell biomechanics, models, and extended studies. Biophys. J. 116(4), 587-594 (2019).

3. Mitchison JM, Swann MM. The mechanical properties of the cell surface. J. Exp. Biol. 31, 443-460 (1954).

4. Garcia-Pelagio KP, Bloch RJ, Ortega A, Gonzalez-Serratos H. Biomechanics of the sarcolemma and costameres in single skeletal muscle fibers from normal and dystrophin-null mice. J. Muscle Res. Cell Motil. 31(5-6), 323-336 (2011).

5. Lovering RM, Michaelson L, Ward CW. Malformed mdx myofibers have normal cytoskeletal architecture yet altered EC coupling and stress-induced Ca2+ signaling. Am. J. Physiol. Cell Physiol. 297(3), C571-C580 (2009).

6. Goodall MH, Ward CW, Pratt SJ, Bloch RJ, Lovering RM. Structural and functional evaluation of branched myofibers lacking intermediate filaments. Am. J. Physiol. Cell Physiol. 303(2), C224-C232 (2012).

7. Head SI. Branched fibres in old dystrophic $\mathrm{mdx}$ muscle are associated with mechanical weakening of the sarcolemma, abnormal Ca2+ transients and a breakdown of Ca2+ homeostasis during fatigue. Exp. Physiol. 95(5), 641-656 (2010).

8. Pichavant C, Pavlath GK. Incidence and severity of myofiber branching with regeneration and aging. Skelet. Muscle 4, 9 (2014).

9. Hernandez-Ochoa EO, Pratt SJ, Garcia-Pelagio KP, Schneider MF, Lovering RM. Disruption of action potential and calcium signaling properties in malformed myofibers from dystrophindeficient mice. Physiol. Rep. 3(4), e12366 (2015).

10. Tarpey MD, Amorese AJ, Balestrieri NP et al. Characterization and utilization of the flexor digitorum brevis for assessing skeletal muscle function. Skelet. Muscle 8(1), 14 (2018).

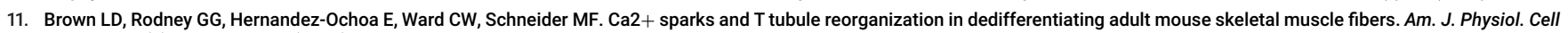
Physiol. 292(3), C1156-C1166 (2007)

12. Rapoport SI. Mechanical properties of the sarcolemma and myoplasm in frog muscle as a function of sarcomere length. J. Gen. Physiol. 59(5), 559-585 (1972).

13. Garcia-Pelagio KP, Muriel J, Neill O et al. Myopathic changes in murine skeletal muscle lacking synemin. Am. J. Physiol Cell Physiol. 308(6), C448-C462 (2015).

14. Solon J, Levental I, Sengupta K, Georges PC, Janmey PA. Fibroblast adaptation and stiffness matching to soft elastic substrates. Biophys. J. 93(12), 4453-4461 (2007).

15. Hall MS, Alisafaei F, Ban E et al. Fibrous nonlinear elasticity enables positive mechanical feedback between cells and ECMs. Proc. Natl Acad. Sci. USA 113(49), 14043-14048 (2016). 